\title{
Comparison of Different Tea Whisk Influence on Bubble Form in Processes of "The Way of Tea"
}

\author{
Tomoko Ota, ${ }^{1, *}$, Wang Zelong ${ }^{2}$, Soutatsu Kanazawa ${ }^{3}$, \\ Yuka Takai ${ }^{4}$, Akihiko Goto ${ }^{4}$, and Hiroyuki Hamada ${ }^{2}$ \\ ${ }^{1}$ Chuo Business Group, Osaka, Japan \\ promotl@gold.ocn.ne.jp \\ ${ }^{2}$ Kyoto Institute of Technology, Kyoto, Japan \\ simon.zelongwang@gmail.com, hhamada@kit.ac.jp \\ ${ }^{3}$ Urasenke Konnichian, Kyoto, Japan \\ kanazawa.kuromon.1352.gentatsu@docomo.ne.jp \\ ${ }^{4}$ Osaka Sangyo University, Kyoto, Japan \\ \{takai,gotoh\}@ise.osaka-sandai.ac.jp
}

\begin{abstract}
In this paper, three kinds of Japanese tea whisks' influence on bubble form in "the way of tea" process were investigated. The bubble form and distribution state by each whisk after $100 \%, 80 \%, 50 \%$ and $30 \%$ of tea making finishing time were recorded and analyzed through numerical processing. In order to verify the quality of tea whisk, three kinds of tea whisks' performance were evaluated and compared during the whole tea making process. Consequently, it can be concluded that "Yabunochi" was the most efficient tea whisk for making a perfect Japanese tea.
\end{abstract}

Keywords: The way of tea, Tea whisk, bubble form, Japanese tea.

\section{Introduction}

The long Japanese ancient culture accumulated a number of traditional artistic activities including "The Way of Tea" ("Chado"), flower arrangement, "Kendo" and so on. Japanese tea ceremony is developed based on "daily after-meal". "The Way of Tea", also called the "Japanese tea ceremony", is a special ceremonial art preparation and presentation of "matcha" (a kind of green tea powder) to entertain the guests, through the tea ceremony people will achieve temperament, improve the cultural quality and aesthetic view. The essence of "The Way of Tea" is meant to demonstrate reverence and respect between host and guest, both of them can truly experience the artistic conception and taste the most primitive taste of green tea during tea-tasting activity and service process with the tallest state of the etiquette.

"The Way of Tea" is consisted of many specific and strict procedures, whose basic skill just only handed over by oral instructions by expert. Furthermore, the spirit of modern Japanese tea ceremony extends to the exterior and interior decoration of tea house. Appreciating the painting and calligraphy decorated in tea house, enjoying the 
gardening design and tea pottery are also the important parts in "The Way of Tea". Among them, using Japanese Tea-whisks stir the tea powder to mixing uniformity and make sure the infusion of the tea leaves combined with the water is the highest technique and important process, which directly affected the taste of tea.

As a an important tool for "The Way of Tea", there appeared some genre of tea whisks with distinguishing shape features and many representative "The Way of Tea" arts masters during the long course of its development. Different genre of tea whisks exhibit has great influence on the development of Japanese tea ceremony. However, the different shapes of tea whisks have different mixing effects and impacts in the whole process of tea making. Basically, tea's mixing uniformity is characteristic by bubble size and distribution attached on the tea surface.

A good tea whisks can mix the green tea power into the hot water with a period of proper time as shown in Fig.1. In order to brewed up a nice cup of tea, masters not only should study and practice for a long time to be an expert and make a high level tea using a user-friendly tea whisk. However, until now the scientific evaluation for the quality of tea whisk is limited. Therefore, it is valuable to conduct some scientific comparison mainstream tea whisks to promote this country cultural treasure and inherit to the next generation effectively.

In this research, 3 types of tea whisks with different shape were investigated as the subject, they were called "Yabunouchi", "Kankyuan", "Ensyu". During "the way of tea" performance, the difference of formed bubbles distribution on the tea surface and temperature variation with different 3 types were inspected and recorded. The characteristic of bubble distribution and the performance of tea whisks were discussed. The bubble form and distribution state after $100 \%, 80 \%, 50 \%$ and $30 \%$ of tea making finishing time described by recording photos were transferred by numerical processing.

As well known, forming process is very critical for the way of tea as a successful tea would depend on the bubble size, distribution and so on. Base on the record of investigation during tea ceremony process, each process's point of degree of mixing and bubble distribution were focused, relationship between timeliness and different tea whisks were extracted and analyzed according to each process.

It is deserved to find that master's action quicker but accurate with the tea whisks of "Yabunouchi". "Yabunouchi" tea whisk made master focus quickly but shift to whisk together green tea powder and water hesitation, which provided a Japanese tea with right temperature and clean tasting for the guests rather than two other tea whisks. It is notify that all type of tea whisk made up the bubble distribution area widely with high foaming degree after $30 \%$ of tea ceremony finishing time. Especially, using "Yabunouchi" tea whisk to make sure that it is easier to make the bubble well-distributed on finished tea surface with less heat loss in less time at last, which showed a strong evidence of a good taste for expert's tea.

In a word, this study was focus on the different tea whisks influence on each tea making process of production. Through numerical processing and analyzing, bubble distribution differences and the characteristic of heat loss on the way. 


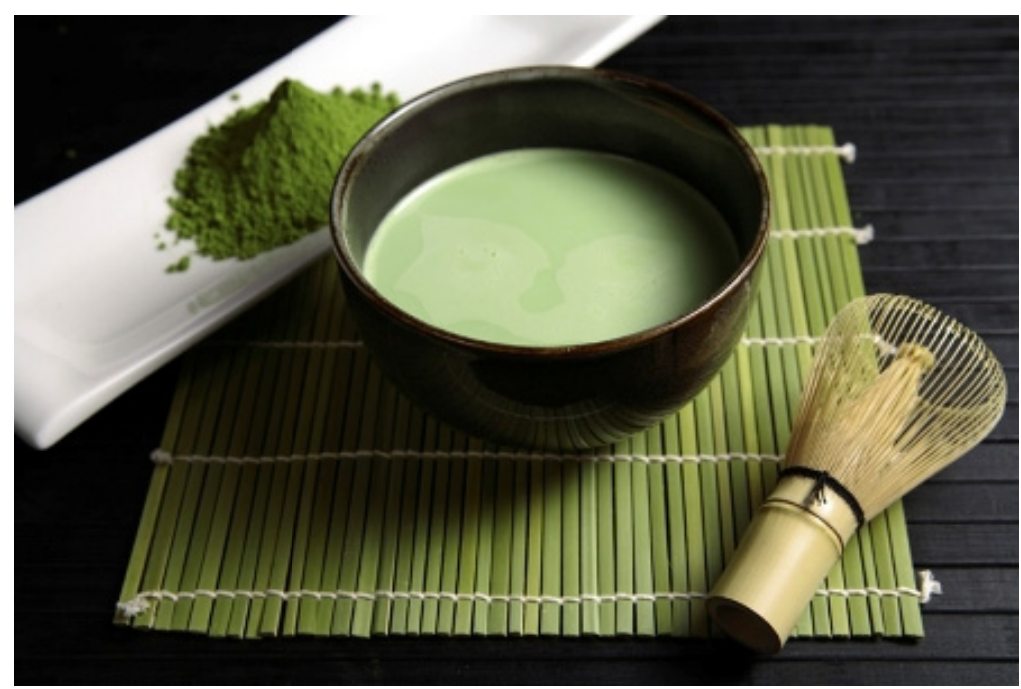

Fig. 1. The way of tea

\section{Experiment}

\subsection{Participants}

A Japanese tea master from Kyoto was employed as the participant. The participant has more than 30 years experience in "the way of tea", who can keep the motion of scooping water and ensure the added water weight in the bowl nearly the same for each tea making process.

\subsection{Subjects}

Three types of Japanese tea whisks were selected for proceeding the experiment called as "Yabunochi", "Kankyuan", "Ensyu", which were the three most popular tea whisks in Japan as shown in Fig.2.

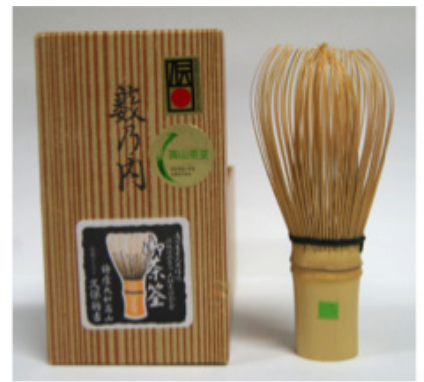

"Yabunochi"

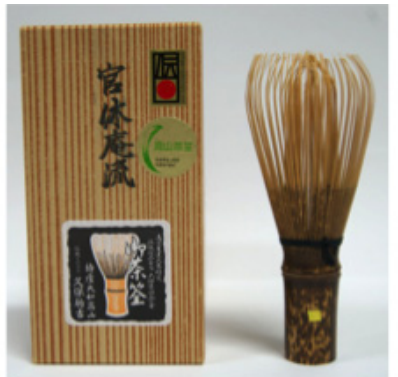

“Kankyuan"

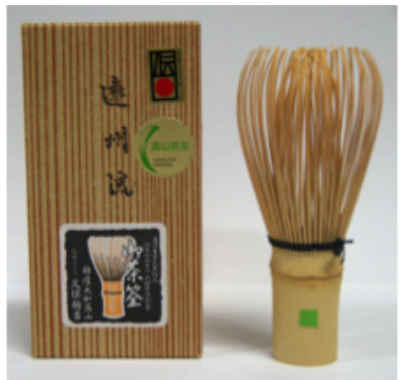

"Ensyu"

Fig. 2. Three types of Japanese tea whisks 


\subsection{Experimental Process}

$1.5 \mathrm{~g}$ of matcha tea power and approximate $56 \mathrm{~g}$ of hot water were dumped into the bowl, and the moisture content of tea was controlled at approximately $97 \%$ steadily. The weight of hot water was illustrated in Tab.1.

Table 1. The weight of the hot water in each trial $(\mathrm{g})$

\begin{tabular}{|c|c|c|c|c|}
\hline & $30 \%$ time & $50 \%$ time & $80 \%$ time & $100 \%$ time \\
\hline Yabunochi & 59.4 & 58.2 & 57.6 & 51.3 \\
\hline Kankyuan & 55.6 & 56.2 & 55.8 & 55.6 \\
\hline Ensyu & 55.2 & 55.9 & 56.1 & 52.7 \\
\hline
\end{tabular}

Four time stages including $100 \%, 80 \%, 50 \%$ and $30 \%$ of tea making finishing time were focused and investigated for the tea made by three kinds of tea whisks. And bubble form and distribution state after $100 \%, 80 \%, 50 \%$ and $30 \%$ of tea making procedure were also recorded and illustrated by single-lens reflex camera (D40x Nikon CO. Ltd). Especially, in order to obtain high-quality photographs a camera device was employed to support and fix the camera as shown in Fig.3.

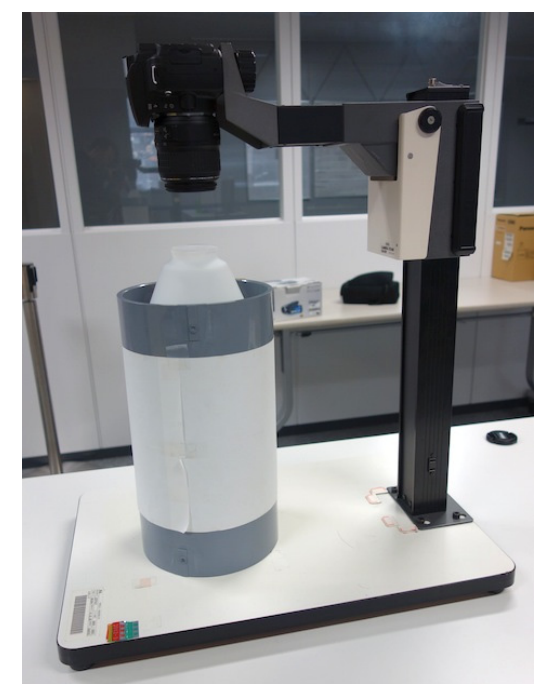

Fig. 3. Camera device

\subsection{Image Processing}

In this research, all the photos were transformed into the same size as the size of the bowl (Diameter: $12.6 \mathrm{~cm}$ ) firstly. Afterwards, circle region located at the center of bowl with 480 pixels were analyzed and transferred by numerical processing from Fig.4(a) to Fig.4(b). It should be mentioned that only bubble forms larger than 


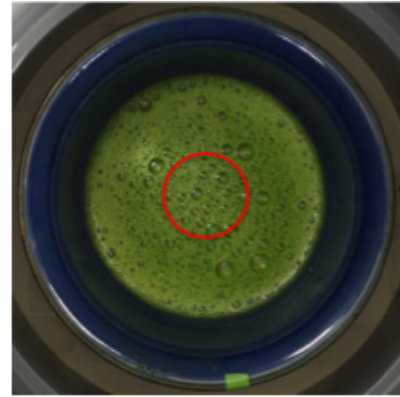

(a) Trim of image

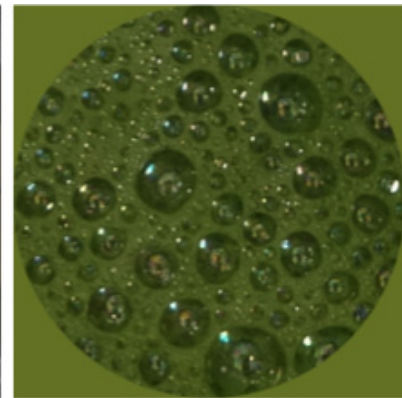

(b) Painting out

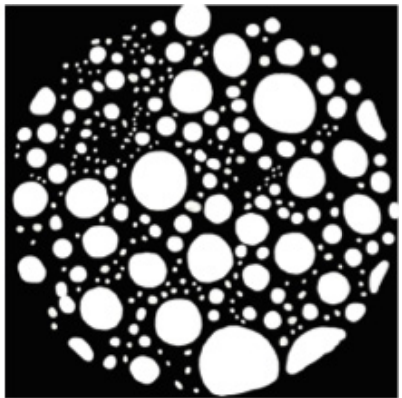

(c) Binarization processing

Fig. 4. Procedure of image processing

$0.03 \mathrm{~mm} 2$ area was marked. Furthermore, marked bubbles were transformed by the binarization processing method into a white and black two colors as shown in Fig.4(c). The outlines of bubble form and bubbles' distribution state were also sketched on the processed image. Finally, the areas of the bubbles were calculated and converted to the area unit.

\section{$3 \quad$ Results and Discussions}

Bubbles' size and the distribution made by three tea whisks in four time stages including $100 \%, 80 \%, 50 \%$ and $30 \%$ of tea making process were presented in Fig.6. The horizontal axis shows the area of the bubble by the logarithm scale and the vertical axis shows the bubble size frequency.

According to Fig.5, it can be found that "Yabunochi" tea whisk is able to produce larger area of bubble at the beginning of tea making procedure as shown in the case of $30 \%$ time. However, comparing with "Yabunochi" performance, the medium sized bubbles were appeared intensively for the same 30\% time case for "Kankyuan" and "Ensyu" as shown in Fig.6 and Fig.7. The areas of bubble produced by three kinds of tea whisks were showed similar distribution in the case of $50 \%$. And it is easy to find that the bubbles existed in the $50 \%$ case was decreased significantly compared with $30 \%$ case. Except for "Yabunouchi" performance, the areas of bubble made by "Kankyuan" and "Ensyu" just showed a slight decreasing trend in the case of 50\%. It is deserved to find that the majority of bubbles existed in the tea which produced by all three kinds of tea whisks are below $1 \mathrm{~mm} 2$ when time stage increased to $80 \%$. There were more points concentrated at the higher position in $80 \%$ case of "Yabunouchi". It was considered that the tea whisk of "Yabunochi" was presented the wider distribution of small bubbles. Additionally, the sizes of the bubbles after mixing by three tea whisks were concentrated in the $0.05 \mathrm{~mm} 2$. And bubbles made by "Kankyuan" and "Ensyu" were almost concentrated in the range of $0.2 \sim 1 \mathrm{~mm} 2$.On the contrast, the bubbles showed smaller size after mixing by applying "Yabunochi" tea whisk. 

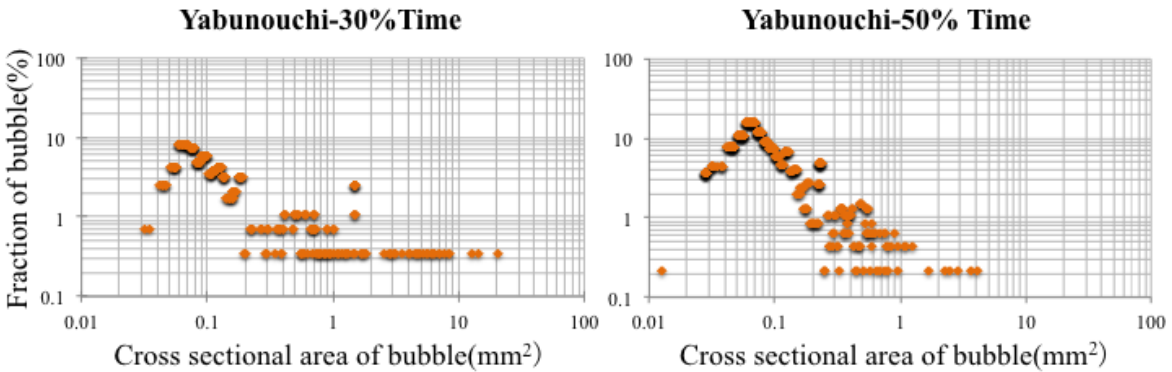

Yabunouchi-80\% Time

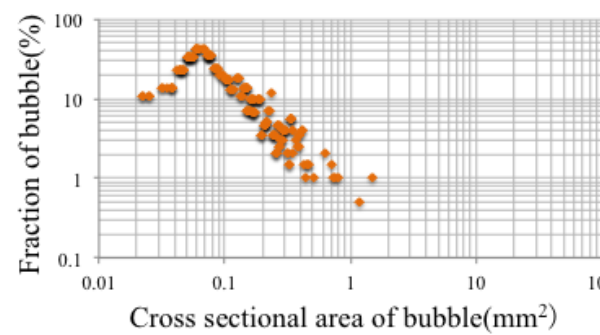

Yabunouchi-100\% Time

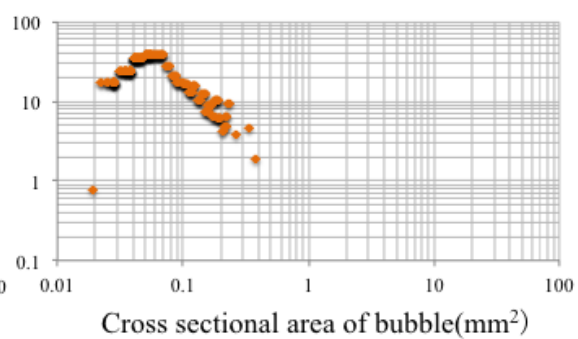

Fig. 5. The form and distribution of bubble in the center of bowl made by "Yabunouchi"

Kankyuan-30\% Time

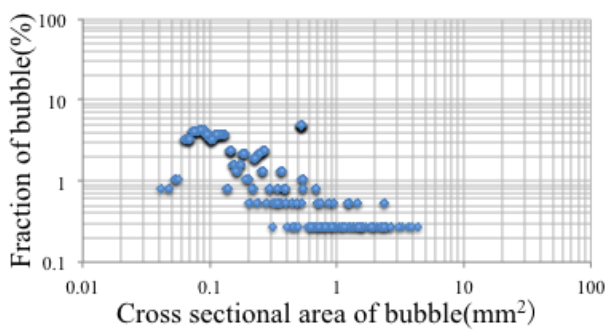

Kankyuan-80\% Time

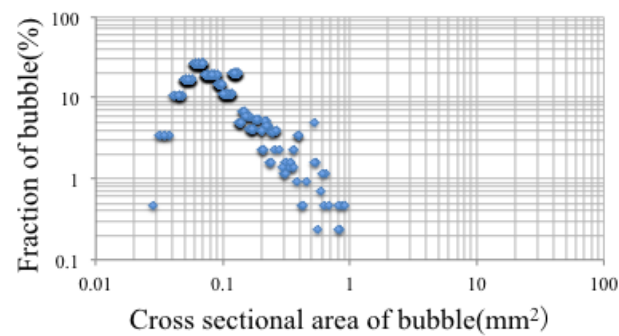

Kankyuan-50\% Time

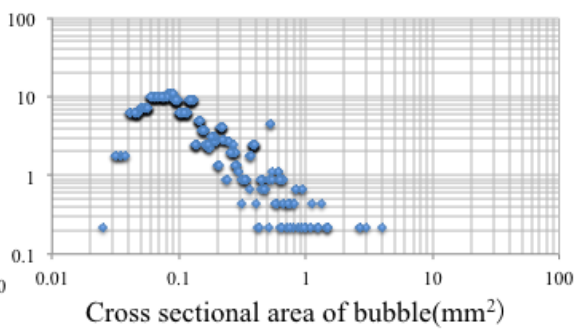

Kankyuan-100\% Time

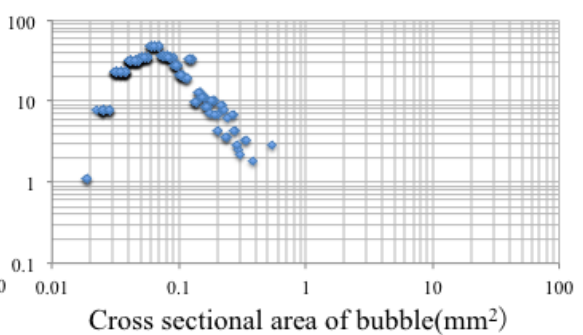

Fig. 6. The form and distribution of bubble in the center of bowl made by "Kankyuan" 
Ensyu-30\% Time
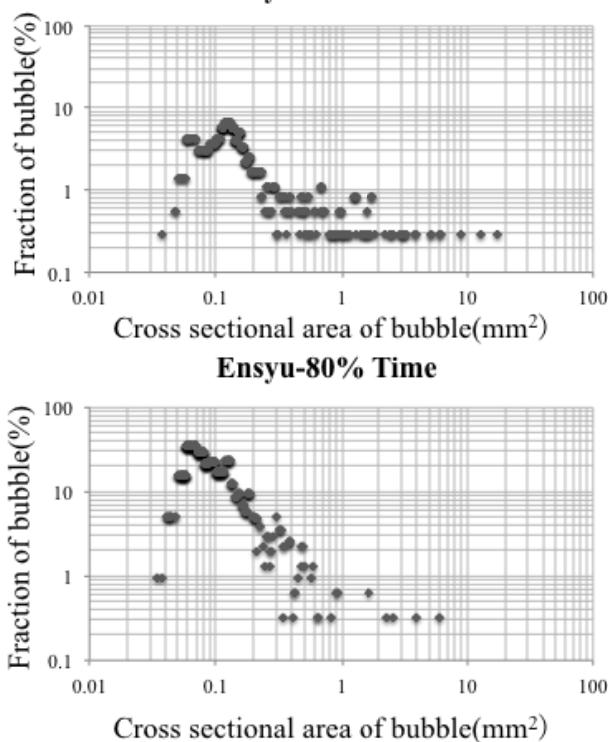

Ensyu-50\% Time

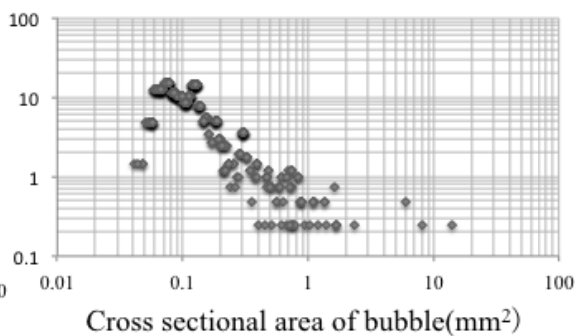

Ensyu-100\% Time

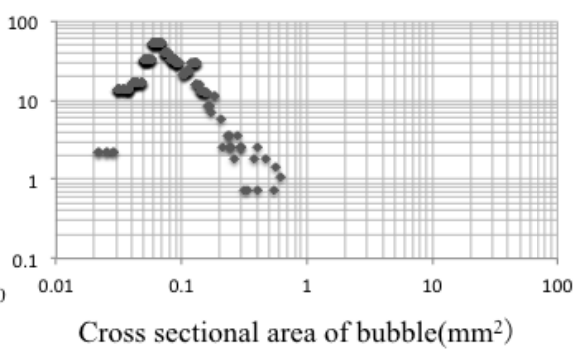

Fig. 7. The form and distribution of bubble in the center of bowl made by "Ensyu"

\section{Conclusions}

In a word, the tea whisk of "Yabunochi" can produce the most widespread bubbles quickly at the beginning of tea making. Afterwards, big bubble size and area existed in previous time stage was changed into smaller one gradually and effectively until the final tea finishing. In other words, it can be concluded that tea powder and hot water can be efficiently mixed together by applying the tea whisk of "Yabunochi".

\section{References}

1. Tujimoto, N., Ichihashi, Y., Iue, M., Ota, T., Hamasaki, K., Nakai, A., Goto, A.: Comparison of bubble forming in a bowl of thin tea between expert and non-expert. In: Proceeding of 11th Japan International SAMPE Symposium \& Exhibition (2009)

2. Goto, A., Endo, A., Narita, C., Takai, Y.: Shimode and, Y., Hamada, H.: Comparison of painting technique of Urushi products between expert and non-expert. In: Advances in Ergonomics in Manufacturing, pp. 160-167 (2012)

3. Aiba, E., Kanazawa, S., Ota, T., Kuroda, K., Takai, Y., Goto, A., Hamada, H.: Developing a System to Assess the Skills of Japanese Way of Tea by Analyzing the Forming Sound: A Case Study. In: Human Factors and Ergonomics Society International meeting (2013) 